\begin{tabular}{|llll}
\hline International Journal of Applied & $\mathbf{A}$ & $\mathbf{I}$ \\
\hline
\end{tabular}

Research-in-Progress

\title{
Path and Position Monitoring Tool for Indoor Robot Application
}

\author{
Ata Jahangir Moshayedi ${ }^{1, *}$, Damayanti Chandrashekar Gharpure ${ }^{1}$ \\ (1) Department of Electronic Science, University of Pune, Pune: 411007, India.
}

\section{Article history:}

Received 18 May 2013 Reviewed 26 May 2013

Revised 28 June 2013

Accepted 30 June 2013

Published 8 July 2013

\begin{abstract}
Robot position monitoring and navigation with ease of use and implementation is a challenge for researchers. The Path and Position Monitoring system (PPMS) is designed for the robot Platform Mokhtar. The path followed by the robot during experimentation, is acquired and displayed graphically using PPMS. The System provides a log of the location $(x, y)$, movement velocity, number of steps for each movement, along with the date and time as a text file. The data can be used to obtain the velocity and movement trajectory of robot for further study. The PPMS can be used for navigation or any other application in robotic studies. The paper presents the design and development of the system and its use in path monitoring of an autonomous wind tracking robot. Various experiments carried out and the results obtained are discussed.
\end{abstract}

Keywords: PPMS, Path and Position Monitoring system, odometry, robot navigation.

\section{Introduction}

Continuous robot position and path monitoring are a requirement in mobile robotics ${ }^{[1-3]}$. In general, Robot position and navigation can be categorized into three types of Global, determining the robot position in absolute or map-referenced terms, Local, based on robot position relative to objects and Personal takes into account the position of various parts, their relative positions for handling objects $^{[2]}$. Amongst mentioned methods personal and local navigation are used more for micro robots ${ }^{[2]}$ but selfpositioning is more in demand in all research ${ }^{[4-6]}$.

Gao and Tseng proposed self-positioning method to measure the distance and orientation of the robot by a sensor, relative to the previous point as it moves toward the target ${ }^{[3]}$.

One of the famous methods in self-positioning is introducing a landmark as a reference point. Lin and Chen proposed two-dimensional (2D) barcode landmark (as reference) which includes the absolute position and has more abilities or error correction automatically ${ }^{[7]}$. For automatic indoor positioning and orientation, the landmarks are placed on the robot and the camera takes pictures of them. Landmark angle and position are acquired, through image segmentation, contour extraction, curves matching characteristics to compute the robot's existing absolute position and heading angle.

Zeungnam et al. studied the 3D self-positioning for a mobile robot with a set of some guide points using vision(stereo camera) and proposed triple guide points

\footnotetext{
* Corresponding author: A.J. Moshayedi ㅁ: +91202569 9841

凶: moshayedi@electronics.unipune.ac.in
}

work well for walking robot with a stereo camera in a laboratory environment ${ }^{[8]}$. On the other hand, for position estimation in addition to Vision based methods, some method like odometry sensor (ultrasonic, infra-red), inertial navigation ${ }^{[3]}$ dead reckoning, etc. are also reported.

The main problem of the above method is due to drift in response with time which affects the accuracy of estimation, especially for ultrasonic and infra-red sensor by the instability in the environment leading to false reflection and echoes ${ }^{[2]}$. Commonly the odometry and inertial navigation methods use active beacons based on artificial landmark recognition, natural landmark recognition, model matching for global position monitoring ${ }^{[5]}$. Orientation and position detection methods based on precise references like the lasers or encoders, in most of the cases are accurate but have the drawback of restriction in object movements ${ }^{[3,9]}$.

Odometry method using encoder for position monitoring is more reliable and recently researchers tried to find a low cost solution ${ }^{[9-11]}$ using the optical mouse. This optical mouse sensor overcomes the common problem with position monitoring ${ }^{[12]}$ :

1. Wheels slipping (by not generating the proper motion of the robot)

2. Crawling (not measuring the robot motion by incremental encoder).

Optical mouse cost and availability, inspire the researcher to do some modification and used it in ROBOCUP matches. The modified optical mouse with the help of microcontroller and SPI interfaces used as a tool for robot positing. The performance of their modified optical mouse on different surfaces has been reported ${ }^{[13]}$. 
On the other hand some researcher attempted use of four computer mouse to get the benefit of accurate positioning $^{[14]}$.

The major objective of this work was to develop a simple, inexpensive, fast system for indoor robot application. The present system gives the ability of monitoring the current robot position as a primary step of navigation based on ball type mouse odometer and providing a simple low cost solution for this problem.

The remainder of the paper is organized as follows. The system design and description are dealt with in Section 2, PPMS design evaluation in Section 3. Finally, paper is concluded in Section 4.

\section{System Design and Description}

The main idea of this work started from the need to develop a small, reliable and low cost solution for monitoring the movement of wind tracker robot designed ${ }^{[15,16]}$. The requirement was to have a tool which can plot the robot movement on-line, graphically and give the position as well as a number of steps, velocity, to study the performance of the tracking algorithm ${ }^{[17-19]}$. In the application of wind tracker the robot is assumed to move on the floor and hence the position is confined to two dimensions $\mathrm{X}$ and $\mathrm{Y}$. A simple, low cost, position monitoring system based on computer mouse has been designed for the same.

\subsection{The Path and Position Monitoring System (PPMS)}

In general, the computer mouse (Fig. 1) is defined as a pointing device which has two rotating rollers (with 90 degrees apart) and attached disk, two sets of send and receive infra-red sensors and a rotating ball (generally made of metal or hard plastic) with ball holder for detecting movement ${ }^{[20]}$.

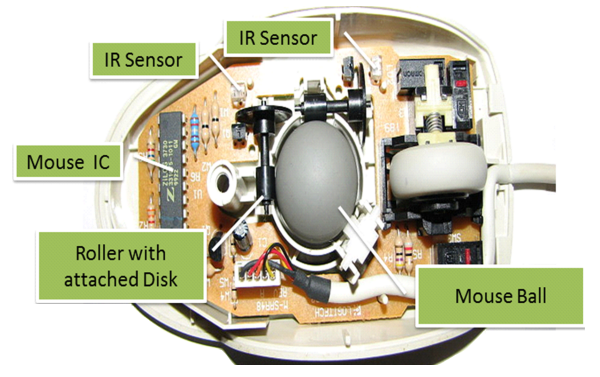

Fig. 1. Ball mouse parts.

\subsubsection{PPMS Hardware}

The computer mouse works same on the encoder wheels and interprets the cursor motion on graphical user interface (GUI) based on light passing between attached disk and infra-red sensors when the roller moves. The encoder signals are converted into motion of the mouse along $\mathrm{X}$ and $\mathrm{Y}$ axes on the screen ${ }^{[6]}$. Considering these features, the same principle was used for the position monitoring system. The PPMS hardware as shown in Fig. 2.

As in Fig. 2 shows, the PPMS hardware uses the computer mouse as an odometric sensing device. Moving the ball in any direction generates the signal which is transferred to the personal computer via a radio frequency module. In order to obtain $(\mathrm{X}, \mathrm{Y})$ position as well as time and velocity, an electrical relay is switched on every second with the help of the accurate timing program running on the ATMEGA 32 microcontroller.

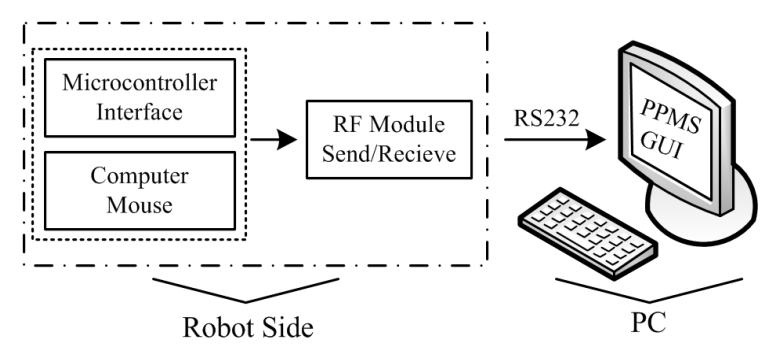

Fig. 2. PPMS block diagram.

This acts as a mouse click in every second to get the robot position accurately. On the other hand to send data from robot platform to computer $\mathrm{RF}$ wireless module CC2500 is used to rectify the limitation of mouse wire. The position data and movement path are monitored by the PPMS software, developed in visual basic (VB6), which is described in the next section.

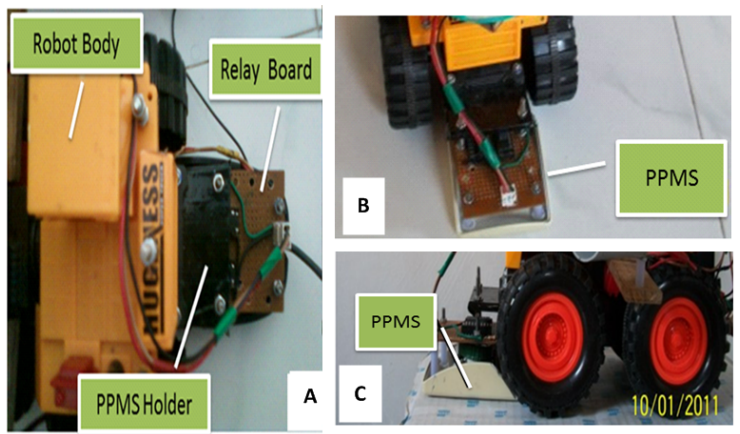

Fig. 3. PPMS hardware on Mokhtar robot platform views; (a) top, (b) front and (c) side view.

Finally for position monitoring the PPMS is mounted between front wheel of robot ${ }^{[12]}$ platform Mokhtar as can be seen in Fig. 3.

\subsubsection{PPMS GUI}

As Fig. 4(a) shows, the PPMS GUI contains two parts of initialisation and path monitoring (Fig. 4(b)).

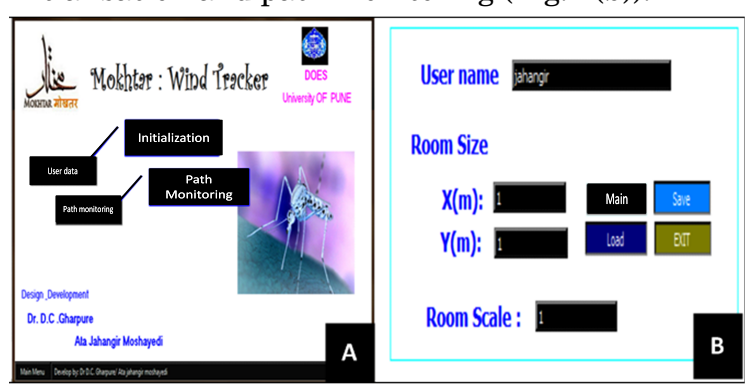

Fig. 4. The PPMS GUI.

The system begins with the initialization and calibration.The parameters set are the user name and the room size (X and Y Dimension of room in meters). Based on the above, the scaling factor named as room scale is calculated and passed to path monitoring module.

The Path monitoring section acquires the position information, logs the location along with time and displays movement of the robot. The robot movement status (sta- 
tionary or moving) indicator, clear option at bottom for cleaning the page, etc. are provided.

At the time of path monitoring the user by entering his name and room dimensions as well as scale can enter on the path monitoring page and start the experiment. By default the initial position of the robot is considered as the origin also by clicking the clear button the cursor will be transferred to the $(0,0)$ location. The location $(\mathrm{X}, \mathrm{Y})$, time, date, and each point of movement is logged into a text file.

\section{PPMS Design Evaluation}

To examine the system performance, the PPMS system was assembled on the robot platform of the Mokhtar ${ }^{[15,16]}$ movement speed of $6 \frac{\mathrm{cm}}{\mathrm{s}}$ and tested in a room with dimension $3 m \times 2.8 m \times 2.5 m^{[16]}$, as shown in Fig. 5.

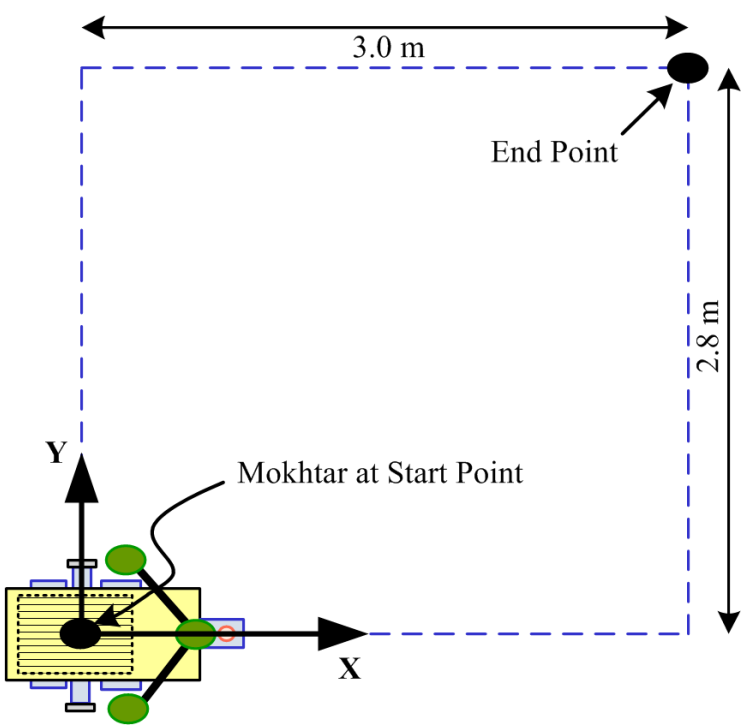

Fig. 5. Experiment environment and Mokhtar robot platform.

The robot was programmed for two different movements.

1. Straight movement for each step of $14 \mathrm{~cm}$ zigzag from a random starting point.

2. Movement with each step of $7 \mathrm{~cm}$, from a random starting point.

In each case the robot movement monitoring was successfully acquired and showed graphically. The result of a path monitoring map for 10 minute is shown in Figures 6 and 7 .

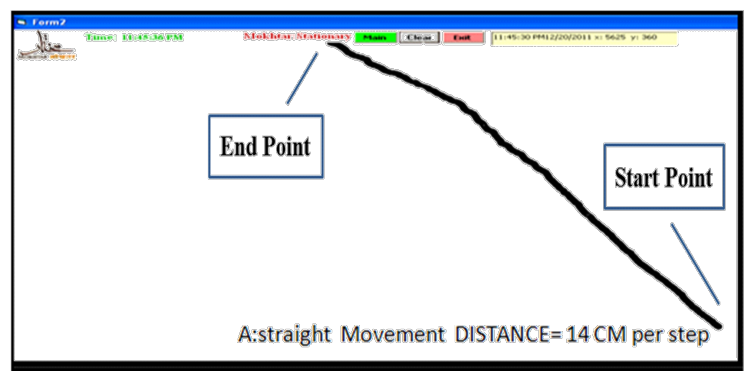

Fig. 6. Robot movement.

Fig. 6 shows the robot path of straight movement and Fig. 7 shows the zigzag path with $7 \mathrm{~cm}$.

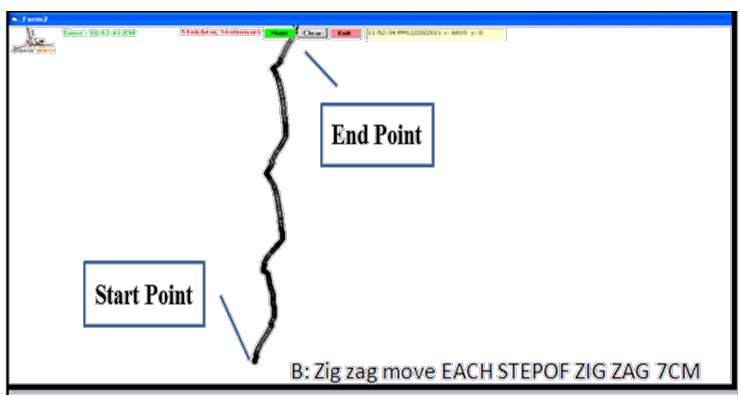

Fig. 7. Robot path monitoring.

\section{Conclusion and Future Works}

With the aim of developing a simple, inexpensive, fast Path and Position Monitoring System for indoor robot application PPMS has been designed. The result and performance on our robot platform shows that the path and position monitoring system (PPMS) is capable of position mapping and monitoring the robot movement. In PPMS, the ability of on-line movement trajectory as well as object location is inbuilt using the computer mouse against the previous work which just obtained the robot position $^{[12]}$. This path monitoring helps to explore the movement of robot under various conditions. Also the data is stored in files which can be accessed through other tools for further analysis. Being cheap and simple permits use of the PPMS on other robot platforms with ease.

By the systematic error in computer mouse ${ }^{[12]}$ the PPMS hardware has some limitation:

1. The system is not able to monitor the turning below 5 degrees.

2 . In the case of surface roughness sometimes the ball movement is not smooth leading to errors. The difficulty can be rectified by the shock absorber or using the optical mouse for darker backgrounds.

One of the major problems by using the computer mouse is the movement limitation on screen. The ball type mouse resolution before any modification in CPI (Counts per inch) is between 100 to $200^{[21]}$. This results in a resolution of $1 \mathrm{~mm}$ in $\mathrm{X}$ direction and $0.5 \mathrm{~mm}$ In Y direction and movement range of $20 \mathrm{~cm}$ in $\mathrm{X}$ direction and $16 \mathrm{~cm}$ in $\mathrm{Y}$ direction. This problem can be solved by making the mouse speed slow by software and increase the thickness of mouse rollers. On the other hand having the extended page in four directions (West south, east, north) with a separate sheet for each direction and adding the previous $\mathrm{X}, \mathrm{Y}$ from the main page also is a solution which is used in this work.

Although to overcome the low sensitivity of optical mouse to the white floor in our lab, the PPMS is designed for a ball type mouse. But due to flexibility in design in the case of the dark texture the ball mouse can be replaced by the optical mouse.

Future work will focus on doing the experiments with PPMS to test wind and plume tracking performance of Mokhtar. Its use for other robot platforms will also be investigated for prediction and estimation of robot position in indoor applications.

\section{REFERENCES}

[1] T. D’Orazio, F. P. Lovergine, M. Ianigro, E. Stella, and A. Distante, "Mobile robot position determination using visual landmarks," Industrial Electronics, IEEE Transac- 
tions on, vol. 41, no. 6, pp. 654-662, 1994.

[2] J. Dixon and O. Henlich, "Mobile robot navigation," Information Systems Engineering Year, Imperial College, vol. 2, pp. $1-10,1997$.

[3] F. Gao and W.-H. Tseng, "Ball mouse," Feb. 2012.

[4] H. Liu, H. Darabi, P. Banerjee, and J. Liu, "Survey of wireless indoor positioning techniques and systems," Systems, Man, and Cybernetics, Part C: Applications and Reviews, IEEE Transactions on, vol. 37, no. 6, pp. 1067-1080, 2007.

[5] M. R. M. Bonarini, A. Matteucci, and M. Restelli, "Dead reckoning for mobile robots using two optical mice," in Proceedings of the 1st International Conference on Informatics in Control, Automation and Robotics (ICINCO), 2004.

[6] F. Claudio, F. Tièche, H. Hügli, C. Facchinetti, F. Tièche, and H. Hügli, "Self-Positioning Robot Navigation Using Ceiling Image Sequences," in ACCV, vol. 95, no. 5, 1995, pp. $5-8$.

[7] G. Y. Lin and X. Chen, "Robot indoor navigation method based on 2D barcode landmark," Applied Mechanics and Materials, vol. 44, pp. 1279-1284, 2011.

[8] B. Zeungnam, K. H. Yeol, Y. Jeongnam, and S. I. Hong, "A Closed Form 3D Self-Positioning algorithm for a mobile robot using vision and guide-marks," 1991.

[9] H. M. Ishak, H. A. Kadir, L. C. Fat, and M. H. A. Wahab, "Autonomous metal detector robot with monitoring system," in Information Technology, 2008. ITSim 2008. International Symposium on, vol. 4. IEEE, 2008, pp. 1-7.

[10] R. Ross, J. Devlin, and S. Wang, "Toward Refocused Optical Mouse Sensors for Outdoor Optical Flow Odometry," Sensors Journal, IEEE, vol. 12, no. 6, pp. 1925-1932, 2012.

[11] M. Cimino and P. R. Pagilla, "Location of optical mouse sensors on mobile robots for odometry," in Robotics and Automation (ICRA), 2010 IEEE International Conference on. IEEE, 2010, pp. 5429-5434

[12] J. Palacin, I. Valganon, and R. Pernia, "The optical mouse for indoor mobile robot odometry measurement," Sensors and Actuators A: Physical, vol. 126, no. 1, pp. 141-147,
2006.

[13] M. Cimino and P. R. Pagilla, "Optimal location of mouse sensors on mobile robots for position sensing," Automatica, vol. 47, no. 10, pp. 2267-2272, 2011.

[14] M. Maier and M. Brandner, "Low-cost optical odometry for wheeled mobile robots," in Instrumentation and Measurement Technology Conference (I2MTC), 2011 IEEE. IEEE, 2011, pp. 1-4.

[15] A. Moshayedi and D. Gharpure, "Design and development of wind tracker," in Computer Applications and Industrial Electronics (ICCAIE), 2011 IEEE International Conference on. Penang: IEEE, 2011, pp. $36-40$

[16] A. J. Moshayedi and D. C. Gharpure, "Development of Position Monitoring system for studying performance of wind tracking algorithms," in Robotics; Proceedings of ROBOTIK 2012; 7th German Conference on. VDE, 2012, pp. 1-4.

[17] C. F. Marques and P. U. Lima, "A localization method for a soccer robot using a vision-based omni-directional sensor," in Robocup 2000: Robot Soccer World Cup IV. Springer, 2001, pp. 96-107.

[18] J. D. Bjerknes, W. Liu, A. F. T. Winfield, C. Melhuish, and C. Lane, "Low cost ultrasonic positioning system for mobile robots," Proceeding of Towards Autonomous Robotic Systems, pp. 107-114, 2007.

[19] N. Sato, K. Kon, H. Fukushima, and F. Matsuno, "Mapbased Navigation Interface for Multiple Rescue Robots," in Safety, Security and Rescue Robotics, 2008. SSRR 2008. IEEE International Workshop on. IEEE, 2008, pp. 152157.

[20] W. Gao, S. Dejima, and S. Kiyono, "Basic study on a selfpositioning technique," in Intelligent Processing Systems, 1997. ICIPS'97. 1997 IEEE International Conference on, vol. 1. IEEE, 1997, pp. 64-69.

[21] I. V. McLoughlin, "Mouse and Tackball," Class note Computer Peripherals course at Nanyang Technological Unitersity, Singapore, pp. 1-15, 2001. [Online]. Available: http://www.lintech.org/comp-per/ 